\title{
Plant growth promoting activities of soil fungi resistant to synthetic fertilizers
}

\author{
Ramasamy Vijayakumar ${ }^{* 1}$, Kasinathan Karthika ${ }^{1}$ and Rajendran Senthilkumar ${ }^{2}$ \\ ${ }^{\prime}$ Department of Microbiology, Government Arts and Science College, Perambalur - 621 107, Tamilnadu, India. \\ ${ }^{2}$ Department of Microbiology, P.G. Extension Centre, Bharathidasan University, Perambalur - 621 107, Tamilnadu, India \\ *CorrespondingauthorEmail: rvijayakumar1979@gmail.com \\ (Submitted on May 5, 2020; Accepted on October 29, 2020)
}

\begin{abstract}
The present study assessed the growth promoting activities of regional soil fungi resistant to chemical fertilizers; besides it also evaluated the resolving sensitivities of the origami optical microscope-Foldscope. Regional agricultural fields derived soil samples were assessed for their physico-chemical parameters and were subsequently processed to isolate the soil fungi. The morphologically identified fungal isolates were also studied microscopically using the Foldscope, a modern microscopic tool. Further, the predominant fungi of the soil were studied for their growth ability on various culture media, different $\mathrm{pH}$ levels and temperature regimes. Impacts of various synthetic fertilizers on the growth of the test fungal isolates were also evaluated. The findings showed that Trichoderma harzianum and Aspergillus niger were the most common isolates from all the sampling sites of Tamilnadu State, India, and were also the most resistant mycoflora against the synthetic fertilizers applied to the agricultural soil. In addition, the plant growth promoting properties of the predominant fungi associated with Vigna mungo crop plant were also described.
\end{abstract}

Keywords: Foldscope, soil fungi, fertilizers tolerance, plant growth promoting activity, Vigna mungo.

\section{INTRODUCTION}

Among the soil microorganisms, fungi play a key role in maintaining the fertility and conversion of complex wastes into the simple nutrients of the soil. They are geographically well distributed and represent the primary components in a broad range of habitats principally in soils rich in decaying vegetation (Bridge and Spooner, 2001).

The growth of soil microorganisms is inhibited by injudicious application of chemicals than any other parameters (Baishya, 2015; Karthika et al., 2019). In this context, there is a genuine need to monitor the fertility of the soil by observing the diversity of soil fungi and all plant growth promoting microorganisms (PGPM), analysis of soil nutrients, and the survivability of soil microbes against chemical fertilizers. This would also facilitate to understand the role of indigenous microbial population in the plant growth promoting activities.

In the present study, the diversity soil fungi was studied using the origami microscope - Foldscope (Cybulski et al., 2014). These were also assessed for their plant growth promoting activities as well as for their toleration capacity against chemical fertilizers.

\section{MATERIALS AND METHODS}

Samples: Soil samples were collected from crop cultivated fields of ten districts of Tamilnadu including Perambalur, Ariyalur, Trichy, Cuddalore, Salem, Nagapattinam, Namakkal, Karur, Thanjavur and Thiruvarur. Sterile polyethylene bags were used to collect the soil samples from a depth of $15 \mathrm{~cm}$, and were transported to the laboratory for further analyses.

Physico-chemical properties: The physico-chemical parameters such as $\mathrm{pH}$, electrical conductivity, organic carbon, organic matter, available nitrogen, phosphorus, potassium, zinc, copper, iron, manganese, calcium, magnesium, sodium and potassium of the soil samples were analyzed by the methods given by Eaton et al. (2005) and Gnanasekaran etal. (2015).
Soil fungi: The soil fungi were isolated by using soil dilution plate method (Hafez, 1982). One gram of each soil sample in $10 \mathrm{~mL}$ of sterile distilled water was suspended and $1 \mathrm{~mL}$ of the suspension of dilutions of $10^{-2}$ and $10^{-3}$ were inoculated on PDA medium added with $1 \%$ streptomycin solution for preventing bacterial growth, before pouring into Petri dishes. The plates were then incubated at $28^{\circ} \mathrm{C}$ for 4-7 days (Ratna Kumar et al., 2017). After incubation, fungal colonies were counted and recorded. The correlation co-efficient analysis between the physico-chemical parameters and fungal population of the soil was studied using SPSS package. Population density was expressed in terms of propagules per gram of soil with dilution factors. The per cent contribution of each isolate was calculated by the following standard formula:

Percentage frequency $=\underline{\text { Total number of propagules of an individual species }} \quad \mathrm{X} 100$ Total number of propagules of all species

Characterization and identification of soil fungi: All the isolated fungal colonies were characterized by the standard methods of Gillman (1957) using lactophenol cotton blue (LCB) technique and were observed under 140X objective of Foldscope (Cybulski et al., 2014). Additionally, identity of the fungi was confirmed through conventional light microscopic observation of the mycelia, conidial structures and spore arrangements. Growth effects of predominant fungal isolates on various culture media, namely PDA, Sabouraud dextrose agar (SDA) and Rose Bengal agar (RBA), temperature at $15,20,25,30$ and $35^{\circ} \mathrm{C}$, and $\mathrm{pH}$ at 5.0, 5.5, 6.0, 6.5 and 7.0 were studied.

Effect of chemical fertilizers on the growth of predominant fungi: The fertilizer tolerance capacity of predominant fungal isolates was studied by the methods of Khattabi et al. (2004). Various concentrations and combinations of chemical fertilizers namely urea, diammonium phosphate and super phosphate were prepared and added into the PDA medium. Then the fungal cultures were inoculated and incubated for 3 days at room temperature. After incubation, radial growth of the fungus was measured, and percentage inhibition of fungal growth 
was calculated by the standard formula:

Percentage of inhibition growth $=\begin{aligned} & \text { Growth in control }- \text { Growth in treatment } \\ & \text { Total number of propagules of all species }\end{aligned} \quad$ X 100

Assessment of mycelia dry weight: Potato dextrose broth was prepared with various combinations of chemical fertilizers (non fertilized control, urea, DAP and urea+super phosphate each $0.5,1.25,2.5$ and $3.25 \mathrm{~g} / \mathrm{mL}$ ) and inoculated with mycelia blocks of test fungi and incubated for 4 days at room temperature. The mycelia mats were collected on 4th day by filtering through the pre-weighed Whatman no.1 filter paper (Kumawat et al., 2016). Then the fungal mycelia were dried in a hot air oven at $50 \pm 2^{\circ} \mathrm{C}$ for $72 \mathrm{~h}$. The actual weight of the dry fungal mycelia was then calculated using the following standard formula:

Weight of drymycelia $=$ weight of filter paper + weight of mycelium - weight of the filter paper

Pot culture experiment: Pot culture experiment was conducted using Vigna mungo plant to study the plant growth promoting activities of Trichoderma harzianum (selected as one of the predominant fungus and known biopesticide), biofertilizers, chemical fertilizers, vermicompost and farm yard manure (FYM) with the combinations of soil + Rhizobium (N2 biofertilizer) + Trichoderma; soil + Phosphobacterium (P biofertilizer) + Trichoderma; soil + Frateuria aurantia (K biofertilizer) + Trichoderma; soil + FYM + Trichoderma; soil + vermicompost + Trichoderma; soil + urea + Trichoderma; soil + super phosphate + Trichoderma and soil + DAP + Trichoderma and soil alone as control. The doses of synthetic fertilizers, vermicompost, FYM and others were applied as recommended by Latha $e t a l$. (2014) and Islam et al. (2017). The seeds were surface disinfected by immersion in $0.5 \%$ bleach solution for $3 \mathrm{~min}$ then were rinsed and washed thrice in sterile distilled water and air-dried in a laminar air flow cabinet (Hajieghrari and Mohammadi, 2016). Ten seeds were buried to 2-3 cm depth in each pot used for treatment and then they were placed under green house condition for 75 days. Three replicates were maintained for all the treatments. The seed germination rate, height of the plant, root and shoot length, numbers of leaves, numbers of branches, number of nodules and number of fruits were measured. The nutrients of soil sample collected from the campus (Kurumbalur, Perambalur district, Tamilnadu) were analyzed after cultivation of black gram by pot culture experiment. Vigna mungo treated soil nutrients data were analyzed using two-way analysis of variance (ANOVA) for mean comparison test at the significance level of $\mathrm{P}<0.05$.

Estimation of leaf chlorophyll content: Chlorophyll content of black gram plant cultivated under pot culture experiment with different growth promoting agents was estimated by Lin et al. (2013). Freeze-dried leaf samples were ground with acetone, centrifuged at $13,000 \mathrm{rpm}$ for $5.0 \mathrm{~min}$. Supernatant was collected and spectrophotometrically measured at 663 and 645 $\mathrm{nm}$ to analyze chlorophyll a and chlorophyll b.

\section{RESULTS}

A total of 1018 fungal colonies were isolated from 38 cultivated field soil samples in ten different districts of Tamilnadu on PDA medium. Maximum fungal propagules $(\mathrm{n}=195)$ were isolated from Ariyalur district, followed by Trichy $(n=132)$, Thanjavur $(n=125)$, Cuddalore $(n=100)$, Perambalur $(n=93)$, Namakkal $(n=92)$, Thiruvarur $(n=90)$, Karur $(n=78)$, Salem $(n=72)$ and Nagapattinam $(n=41)$ (Table 1). Among 1018 propagules, 8 morphologically distinguished fungal isolates were identified. Trichoderma harzianum with 231 propagules and percentage frequency of $22.69 \%$ was predominant, followed by Aspergillus niger (225 propagules; $22.1 \%)$, A. flavus (133 propagules; $13.06 \%$ ), A. fumigatus (127 propagules; 12.47\%), Rhizopus sp. (116 propagules; 11.39\%), Aspergillus terreus (86 propagules; 8.44\%), Fusarium oxysporum (66 propagules; 6.48\%) and Cephalosporium sp. (34 propagules; 3.33\%) (Table 1). Fungal isolates were identified based on their colony morphology and foldscope observations (Fig. 1). Further, identity of the fungi was confirmed through conventional
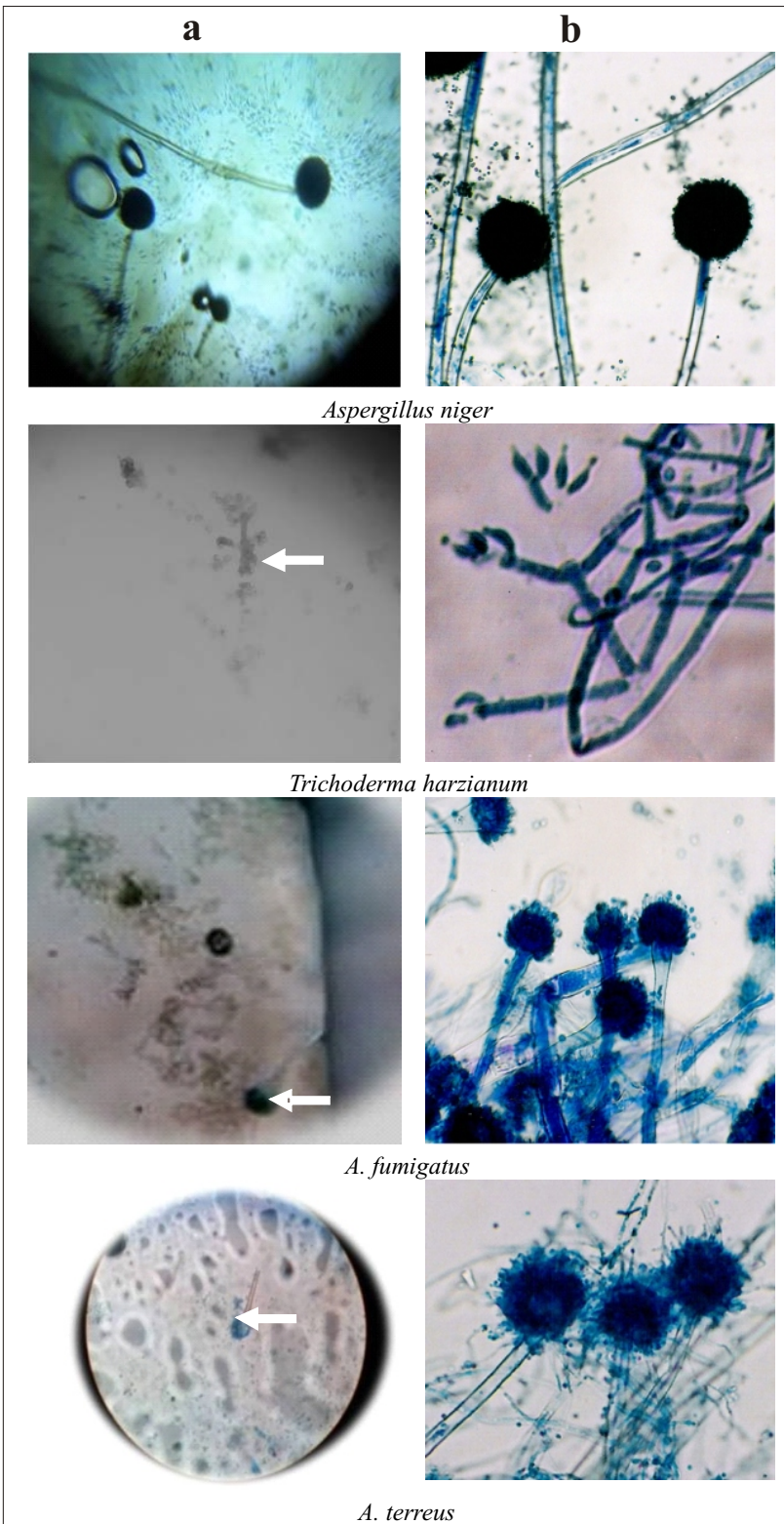

Fig. 1. Microscopic morphologies of the test soil fungal isolates (a) Foldscope (b) Light Microscope 
Table 1. Frequency of mycoflora in different sampling stations.

\begin{tabular}{|c|c|c|c|c|c|c|c|c|c|c|}
\hline $\begin{array}{l}\text { Sampling } \\
\text { stations }\end{array}$ & 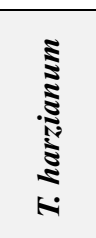 & 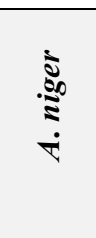 & $\underset{⿱ 亠 乂}{\tilde{\Sigma}}$ & 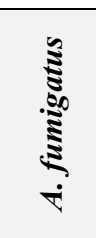 & 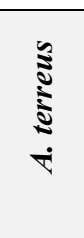 & 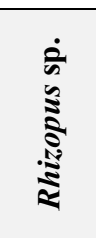 & 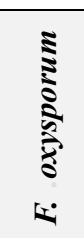 & 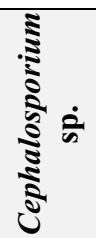 & 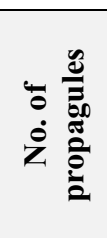 & 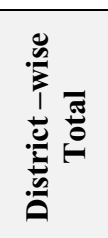 \\
\hline $\begin{array}{l}\text { Perambalur Dt. } \\
\text { Perambalur }\end{array}$ & - & 7 & 5 & 4 & 2 & - & 3 & - & 21 & \\
\hline Alathur & 5 & 6 & 4 & 2 & 1 & 3 & 3 & 2 & 26 & 93 \\
\hline Kunnam & 6 & 5 & 2 & 3 & 0 & 3 & 4 & - & 23 & \\
\hline Veppanthattai & - & 6 & 3 & 3 & 2 & 4 & 2 & 3 & 23 & \\
\hline $\begin{array}{l}\text { Ariyalur Dt. } \\
\text { Ariyalur }\end{array}$ & 6 & 5 & 4 & 8 & - & 7 & 3 & - & 33 & \\
\hline Udayarpalayam & 6 & 5 & 6 & 5 & 4 & 7 & - & 3 & 36 & \\
\hline Sendurai & 8 & 6 & 4 & 4 & 2 & 6 & - & 3 & 33 & 195 \\
\hline Andimadam & 10 & 6 & 7 & 8 & 4 & 7 & 3 & 2 & 47 & \\
\hline Paluvur & 11 & 7 & 6 & 5 & 5 & 6 & 2 & 4 & 46 & \\
\hline $\begin{array}{l}\text { Trichy Dt. } \\
\text { Thuraiyur }\end{array}$ & 4 & 6 & 4 & 4 & 2 & - & - & - & 20 & \\
\hline Musiri & 5 & 6 & - & 4 & 3 & 5 & 3 & - & 26 & \\
\hline Lalgudi & 5 & 7 & 4 & 3 & 3 & 4 & 2 & 2 & 30 & 132 \\
\hline Pullambadi & 5 & 4 & 3 & - & 2 & 3 & - & - & 17 & \\
\hline Thottiyam & - & 4 & 4 & 3 & - & 2 & - & - & 13 & \\
\hline Pachai Hills & 8 & 6 & 5 & 7 & - & - & - & - & 26 & \\
\hline $\begin{array}{l}\text { Cuddalore Dt. } \\
\text { Veppur }\end{array}$ & 7 & 8 & 5 & 3 & 3 & 4 & - & - & 30 & \\
\hline Pennadam & 10 & 10 & 3 & - & 2 & - & - & - & 25 & 100 \\
\hline Virudhachalam & 8 & 6 & 2 & 4 & 2 & 1 & 2 & - & 25 & \\
\hline Thittakudi & 6 & 4 & - & 2 & 3 & 3 & 2 & - & 20 & \\
\hline $\begin{array}{l}\text { Salem Dt. } \\
\text { Gangavalli }\end{array}$ & 6 & 3 & 2 & 3 & - & 2 & 2 & - & 18 & \\
\hline Athur & 5 & 8 & 4 & 4 & - & 3 & 2 & 2 & 28 & 72 \\
\hline Thalaivasal & 4 & 8 & 5 & 3 & 2 & 2 & 2 & - & 26 & \\
\hline $\begin{array}{l}\text { Nagapatinam Dt. } \\
\text { Mayiladuthurai }\end{array}$ & 4 & 5 & 5 & 3 & 3 & 2 & 3 & - & 25 & 41 \\
\hline Tharangambadi & - & 3 & - & 3 & 3 & 4 & 3 & - & 16 & \\
\hline $\begin{array}{l}\text { Namakkal Dt. } \\
\text { Namakkal }\end{array}$ & 4 & 5 & 4 & 3 & 4 & 3 & 2 & 1 & 26 & \\
\hline Rasipuram & 8 & 2 & 3 & - & 3 & 2 & - & - & 18 & 92 \\
\hline Mohanur & 8 & 8 & 3 & 2 & 2 & - & 3 & 2 & 28 & \\
\hline Paramathi & 7 & 5 & - & 3 & 3 & 2 & - & - & 20 & \\
\hline Karur Dt.Karur & 8 & 5 & 2 & - & 3 & 4 & - & - & 22 & \\
\hline Krishnarayapuram & 9 & 7 & 4 & 3 & - & 2 & 2 & - & 27 & 78 \\
\hline Kulithalai & 5 & 8 & 4 & 3 & 2 & 4 & 3 & - & 29 & \\
\hline $\begin{array}{l}\text { Thanjavur Dt. } \\
\text { Thanjavur }\end{array}$ & 8 & 3 & 5 & 4 & 2 & 4 & 3 & 2 & 31 & \\
\hline Orathanadu & 5 & 10 & 4 & 3 & 4 & 5 & 2 & 3 & 36 & 125 \\
\hline Thiruvaiyaru & 8 & 5 & - & - & 3 & 4 & 3 & 2 & 25 & \\
\hline Pattukottai & 7 & 8 & 4 & 4 & 4 & 3 & 3 & - & 33 & \\
\hline $\begin{array}{l}\text { Thiruvarur Dt. } \\
\text { Nannilam }\end{array}$ & 10 & 8 & 5 & 5 & 3 & - & 2 & - & 33 & \\
\hline Muthupet & 6 & 5 & 6 & 6 & 3 & 2 & - & 3 & 31 & 90 \\
\hline Mannargudi & 9 & 5 & 2 & 3 & 2 & 3 & 2 & - & 26 & \\
\hline Total & 231 & 225 & 133 & 127 & 86 & 116 & 66 & 34 & \multirow{2}{*}{\multicolumn{2}{|c|}{$\begin{array}{l}1018 \\
\text { Average } * 26.78 \%\end{array}$}} \\
\hline$\%$ frequency & 22.69 & 22.10 & 13.06 & 12.47 & 8.44 & 11.39 & 6.48 & 3.33 & & \\
\hline
\end{tabular}


Table 2. Physico-chemical properties of soil samples.

\begin{tabular}{|c|c|c|c|c|c|c|c|c|c|c|c|}
\hline \multirow[b]{2}{*}{ Sampling station } & \multicolumn{11}{|c|}{ Parameters } \\
\hline & $\mathbf{p H}$ & $\frac{\mathbf{E C}_{-1}}{\left(\mathrm{dSm}^{-1}\right)}$ & $\begin{array}{l}\mathbf{C a C O}_{3} \\
(\mathrm{mg} / \mathrm{kg})\end{array}$ & $\underset{(\mathrm{kg} / \mathrm{ha})}{\mathbf{N}}$ & $\underset{(\mathrm{kg} / \mathrm{ha})}{\mathbf{P}}$ & $\underset{(\mathrm{kg} / \mathrm{ha})}{\mathbf{K}}$ & $\begin{array}{c}\mathbf{F e} \\
(\mathrm{ppm})\end{array}$ & $\begin{array}{c}\text { Mn } \\
(\mathrm{ppm})\end{array}$ & $\begin{array}{c}\mathbf{Z n} \\
(\mathrm{ppm})\end{array}$ & $\begin{array}{c}\mathbf{C u} \\
(\mathrm{ppm})\end{array}$ & $\begin{array}{l}\mathrm{OC} \\
(\%)\end{array}$ \\
\hline $\begin{array}{l}\text { Perambalur Dt. } \\
\text { Perambalur }\end{array}$ & 7.7 & 0.40 & High & 59.6 & 11.3 & 336 & 2.60 & 2.30 & 0.96 & 0.74 & 0.33 \\
\hline Alathur & 7.8 & 1.55 & High & 62.8 & 12.5 & 193.5 & 2.75 & 1.97 & 0.87 & 0.84 & 0.45 \\
\hline Kunnam & 7.94 & 0.36 & High & 59.6 & 8.75 & 343 & 2.90 & 2.20 & 0.94 & 0.84 & 0.40 \\
\hline Veppanthattai & 8.07 & 0.20 & High & 56.4 & 6.25 & 387 & 2.75 & 2.15 & 0.80 & 0.94 & 0.39 \\
\hline Pachai Hills & 7.32 & 1.22 & Medium & 65.7 & 13.8 & 288 & 1.98 & 1.88 & 0.97 & 0.88 & 0.40 \\
\hline $\begin{array}{l}\text { Trichy Dt. } \\
\text { Thuraiyur }\end{array}$ & 7.4 & 1.6 & Medium & 58.1 & 12.5 & 297 & 2.3 & 2.1 & 0.89 & 0.79 & 0.56 \\
\hline Musiri & 7.2 & 1.42 & Medium & 70.2 & 11.7 & 312 & 1.89 & 2.31 & 0.91 & 0.76 & 0.62 \\
\hline Lalgudi & & & High & 68.8 & 7.27 & 287.2 & 1.80 & 1.96 & 0.93 & 0.67 & 0.48 \\
\hline Pullambadi & 7.9 & 1.15 & High & 58.2 & 8.97 & 317 & 2.17 & 1.87 & 0.82 & 0.68 & 0.51 \\
\hline Thottiyam & 7.0 & 1.28 & Medium & 63.2 & 9.7 & 326 & 2.89 & 2.03 & 0.87 & $0.91 \mathrm{~s}$ & 0.64 \\
\hline $\begin{array}{l}\text { Cuddalore Dt. } \\
\text { Veppur }\end{array}$ & 6.9 & 1.32 & High & 74.9 & 9.8 & 298.7 & 2.10 & 1.87 & 0.94 & 0.88 & 0.45 \\
\hline Pennadam & 7.2 & 1.45 & High & 72.1 & 10.7 & 287.1 & 1.98 & 1.93 & 0.77 & 0.80 & 0.56 \\
\hline Virudhachalam & 7.3 & 1.29 & High & 70.2 & 11.5 & 269 & 1.87 & 2.41 & 0.81 & 0.79 & 0.63 \\
\hline Thittakudi & 7.5 & 1.34 & High & 71.7 & 13.1 & 287 & 1.89 & 2.54 & 0.73 & 0.81 & 0.48 \\
\hline $\begin{array}{l}\text { Ariyalur Dt. } \\
\text { Ariyalur }\end{array}$ & 8.24 & 1.32 & High & 58.6 & 12.1 & 291.7 & 2.87 & 2.18 & 0.98 & 0.98 & 0.30 \\
\hline Udayarpalayam & 8.02 & 1.82 & High & 61.7 & 12.9 & 307.8 & 2.58 & 2.08 & 0.94 & 0.85 & 0.32 \\
\hline Sendurai & 8.31 & 1.27 & High & 60.3 & 11.7 & 312 & 2.78 & 2.11 & 0.88 & 0.78 & 0.38 \\
\hline Andimadam & 8.08 & 1.30 & High & 61.4 & 10.9 & 327.8 & 2.45 & 2.31 & 0.96 & 0.82 & 0.35 \\
\hline Paluvur & 7.80 & 1.23 & High & 59.7 & 10.5 & 299.2 & 2.12 & 1.93 & 0.82 & 0.79 & 0.41 \\
\hline $\begin{array}{l}\text { Salem Dt. } \\
\text { Gangavalli }\end{array}$ & 8.1 & 0.76 & High & 60.8 & 12.7 & 324.9 & 1.58 & 2.30 & 0.89 & 0.92 & 0.52 \\
\hline Athur & 8.3 & 0.90 & High & 58.3 & 11.9 & 318.7 & 3.87 & 3.2 & 1.05 & 0.99 & 0.32 \\
\hline Thalaivasal & 8.4 & 0.46 & High & 59.2 & 11.2 & 268.8 & 4.6 & 2.98 & 0.95 & 0.91 & 0.62 \\
\hline $\begin{array}{l}\text { Nagapattinam Dt. } \\
\text { Mayiladuthurai }\end{array}$ & 7.7 & 0.78 & High & 53.2 & 8.2 & 331.0 & 3.28 & 3.11 & 0.66 & 0.85 & 0.58 \\
\hline Tharangambadi & 8.2 & 1.21 & High & 55.4 & 9.7 & 311.8 & 3.11 & 2.45 & 0.82 & 0.90 & 0.60 \\
\hline $\begin{array}{l}\text { Namakkal Dt. } \\
\text { Namakkal }\end{array}$ & 7.2 & 1.25 & High & 59.1 & 10.7 & 308.0 & 4.88 & 1.98 & 0.93 & 0.88 & 0.38 \\
\hline Rasipuram & 7.3 & 1.32 & High & 60.2 & 11.2 & 266.0 & 3.89 & 2.31 & 0.84 & 085 & 0.45 \\
\hline Mohanur & 7.4 & 1.14 & High & 61.7 & 9.1 & 263.7 & 3.2 & 2.85 & 0.87 & 0.76 & 0.47 \\
\hline Paramathi & 7.0 & 1.38 & & 65.2 & 9.6 & 254.0 & 4.2 & 3.02 & 0.79 & 0.82 & 0.51 \\
\hline $\begin{array}{l}\text { Karur Dt. } \\
\text { Karur }\end{array}$ & 7.0 & 1.41 & Medium & 66.1 & 10.1 & 189.2 & 3.1 & 2.55 & 0.91 & 0.76 & 0.46 \\
\hline Krishnarayapuram & 7.6 & 1.39 & High & 62.3 & 8.9 & 198.2 & 3.22 & 2.41 & 0.82 & 0.81 & 0.52 \\
\hline Kulithalai & 7.7 & 1.27 & Medium & 71.0 & 9.5 & 200.7 & 4.5 & 2.31 & 0.81 & 0.72 & 0.66 \\
\hline $\begin{array}{l}\text { ThanjavurDt. } \\
\text { Thanjavur }\end{array}$ & 7.2 & 7.4 & Medium & 88.3 & 7.6 & 285.2 & 6.5 & 3.62 & 0.91 & 0.82 & 0.53 \\
\hline Orathanadu & 7.5 & 1.96 & Medium & 90.2 & 8.2 & 263.1 & 6.85 & 3.69 & 0.86 & 0.78 & 0.39 \\
\hline Thiruvaiyaru & 7.6 & 1.60 & Medium & 80.2 & 10.4 & 234.2 & 5.6 & 3.48 & 0.90 & 0.85 & 0.44 \\
\hline Pattukottai & 7.8 & 1.85 & High & 95.1 & 9.8 & 221.2 & 6.33 & 3.71 & 0.89 & 0.78 & 0.37 \\
\hline $\begin{array}{l}\text { Thiruvarur Dt. } \\
\text { Nannilam }\end{array}$ & 7.1 & 1.62 & Medium & 79.2 & 10.2 & 228.9 & 4.5 & 2.89 & 0.75 & 0.75 & 0.58 \\
\hline Muthupet & 8.1 & 1.69 & High & 95.7 & 13.7 & 296.3 & 7.66 & 3.09 & 0.94 & 0.98 & 0.31 \\
\hline Mannargudi & 7.6 & 0.81 & Medium & 82.0 & 11.2 & 255.8 & 5.6 & 3.21 & 0.91 & 0.68 & 0.41 \\
\hline
\end{tabular}


light microscope by observing mycelia and conidial structures and spore arrangement. The studied physicochemical properties of the soil samples are presented in table 2. Statistically, relationships between the soil fungi and physico-chemical properties were studied by correlation coefficient analysis and it was found that there was no significant positive correlation co-efficient between the physico-chemical properties of soil and total fungal population, whereas, significant positive and negative correlation co-efficient were observed between the nutrients of the soil (Table 3 ).

Fertilizer tolerance capacity of the predominant $A$. niger and T. harzianum were studied against urea, di-ammonium phosphate (DAP) and super phosphate, and it was found that the radial growth of both the test fungi was significantly affected by urea at $8 \mathrm{~g} / \mathrm{L}(80.3 \%$ and $75.7 \%)$ followed by the combination of DAP and super phosphate and DAP (72.7\% and $63.3 \%$ and $70.5 \%$ and $60.3 \%$ at $8 \mathrm{~g} / \mathrm{L}$ ) (Fig. 2a-c). Fertilizer tolerance ability of the test fungi was further determined by the measurement of mycelial dry weight, and it was found that the mycelia dry weight was reduced in the media with fertilizers when compared to control. Among the three chemical fertilizers tested, the mycelia dry weight was significantly reduced at higher concentration of urea and super phosphate combination $(0.8 \mathrm{~g}$ at $3.75 \mathrm{~g} / 100 \mathrm{~mL}$ and 1.0 $\mathrm{g}$ at $3.75 \mathrm{~g} / \mathrm{L}$ for $A$. niger and T. harzianum, respectively) (Fig. 3). Both the predominant fungi could be grown well at $\mathrm{pH} 5.5$, temperature at $30{ }^{\circ} \mathrm{C}$ on PDA medium during $4^{\text {th }}$ day in comparison to the other media and culture conditions tested.

Effect of chemicals as well as biofertilizers and FYM and vermicompost on the morphometric study of Vigna mungo was also studied. All the test seeds $(100 \%)$ were germinated in all the twenty-four treatments including control. The tallest $(39 \pm 0.52 \mathrm{~cm})$ black gram plant was observed in the pot treated with Rhizobium sp. (T12), maximum root length $(15 \pm 0.5 \mathrm{~cm})$ was observed in the plant treated with the combination of all the three biofertilizers (T20), namely Rhizobium sp., Phosphobacterium sp. and Frateuria aurantia. Maximum $(25.5 \pm 0.50 \mathrm{~cm})$ shoot length was observed in both $\mathrm{T} 3$ and $\mathrm{T} 21$ treatments. Both height and root length of the plant were highly influenced by the combination of NPK biofertilizers, whereas the length of shoot was induced by the T1-T3, T12, T14 and combination of both chemical and biofertilizers.
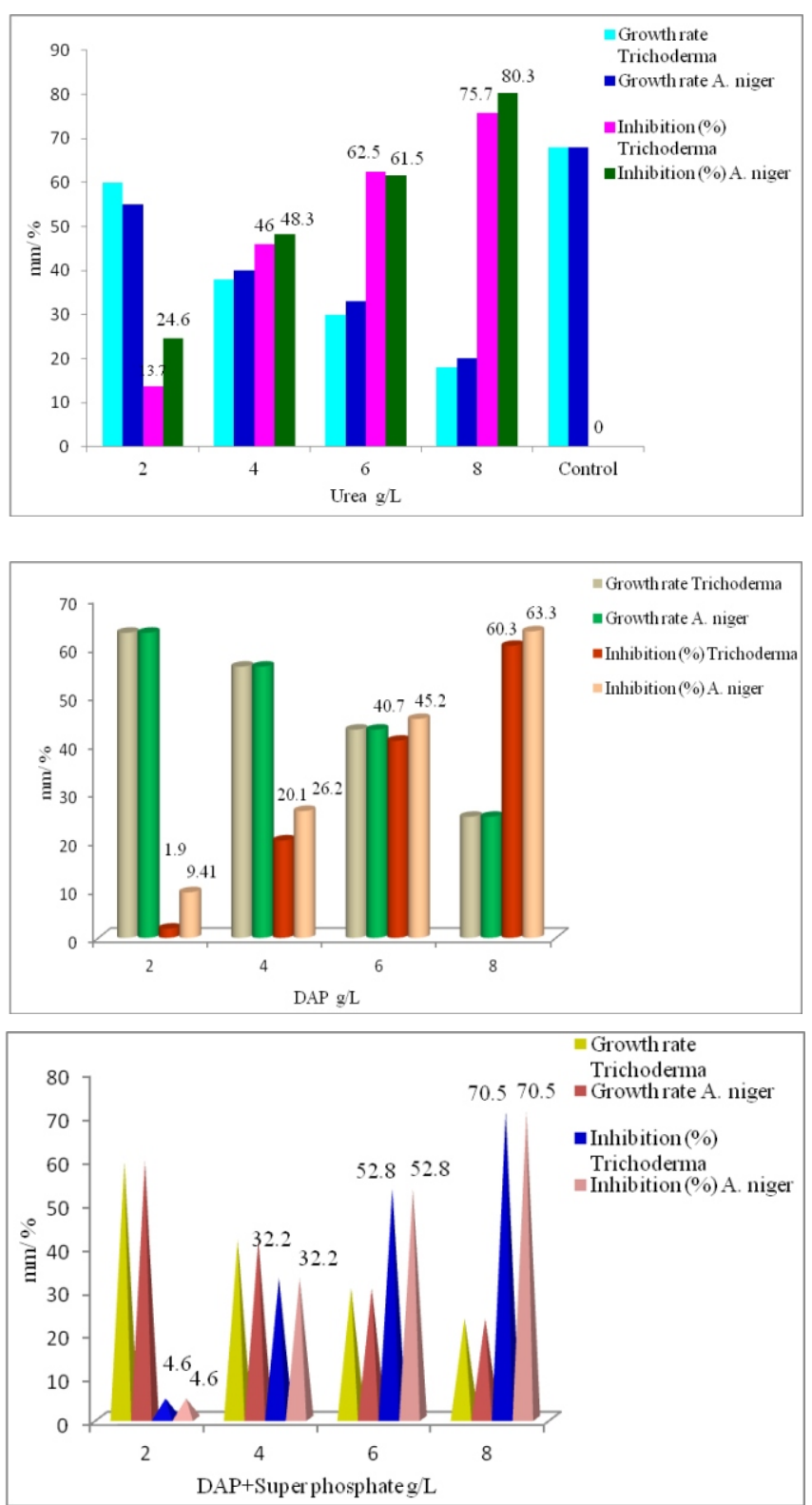

Fig. 2. Effect of various chemical pesticides on the radial growth of Trichoderma harzianum and Aspergillus niger a) Urea; b) DAP; c) DAP+ Super phosphate

Table 3. Correlation co-efficient between physico-chemical properties of soil samples and total fungal colonies

\begin{tabular}{|c|c|c|c|c|c|c|c|c|c|c|}
\hline & TFC & $\mathbf{P h}$ & $\mathbf{N}$ & $\mathbf{P}$ & $\mathbf{K}$ & $\mathbf{F e}$ & Mn & $\mathbf{Z n}$ & $\mathbf{C u}$ & $\mathrm{OC}$ \\
\hline TFC & 1 & & & & & & & & & \\
\hline pH & -0.3601 & 1 & & & & & & & & \\
\hline $\mathbf{N}$ & -0.3682 & -0.6815 & 1 & & & & & & & \\
\hline $\mathbf{P}$ & 0.02432 & $-0.9074^{* *}$ & $0.9174^{* *}$ & 1 & & & & & & \\
\hline $\mathbf{K}$ & 0.3590 & 0.5225 & $-0.9403^{*}$ & $-0.8297^{*}$ & 1 & & & & & \\
\hline $\mathrm{Fe}$ & $-0.9205^{* *}$ & 0.6058 & 0 & -0.3743 & 0.0340 & 1 & & & & \\
\hline Mn & 0.5710 & -0.1424 & -0.5317 & -0.2701 & $0.7586^{*}$ & -0.2954 & 1 & & & \\
\hline $\mathbf{Z n}$ & 0.0774 & -0.7104 & 0.3928 & 0.5255 & -0.0642 & -0.1122 & 0.5487 & 1 & & \\
\hline $\mathbf{C u}$ & -0.3682 & $0.9339^{* *}$ & -0.5 & $-0.7412^{*}$ & 0.2478 & 0.5 & -0.4430 & $-0.8979^{* * *}$ & 1 & \\
\hline $\mathrm{OC}$ & $-0.8013^{* *}$ & 0.2772 & 0.4974 & 0.1521 & -0.6662 & 0.5803 & $-0.9477^{*}$ & -0.4582 & 0.4974 & 1 \\
\hline
\end{tabular}




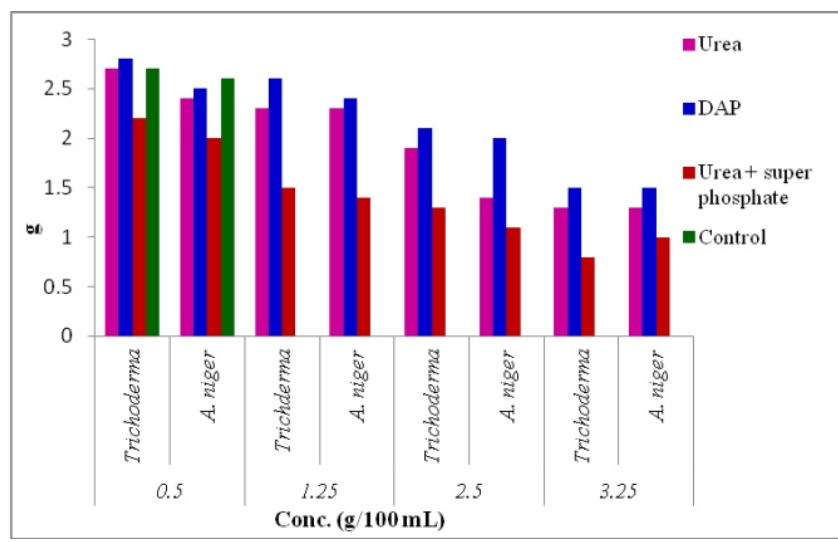

Fig. 3. Effect of various fertilizers on the mycelial dry weights of Trichoderma harzianum and Aspergillus niger

Number of leaves $(n=10)$, branches $(n=6)$, nodules $(n=28 \pm 3.21)$ and fruits $(n=5)$ were recorded in the plant treated using biofertilzers, particularly nitrogen based biofertilizer Rhizobium (T12) (Table 4). Similarly, maximum contents of both chlorophyll a $\left(19.28 \mathrm{mg} / \mathrm{m}^{2}\right)$ and b $(28.21$ $\mathrm{mg} / \mathrm{m}^{2}$ ) were recorded in T12. Chlorophyll b content was

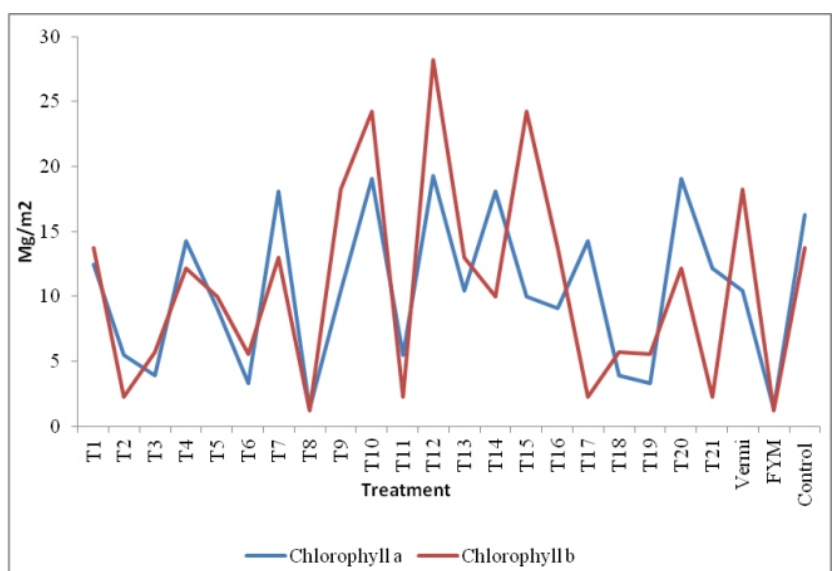

Fig.4. Effect of various treatments on chlorophyll content of Vigna mungo

higher than chlorophyll a in almost all the treatments (Fig. 4). After treatment, EC content was less than $1.0 \mathrm{dSm}^{-1}$ in all the biofertilizers as well as chemical fertilizers treated soil. Contents of N, P, K, Fe and Mn were slightly increased due to the introduction of NPK based biofertilizers. Whereas heavy

Table 4. Effect of various treatments on the morphometric parameters of Vigna mungo

\begin{tabular}{|c|c|c|c|c|c|c|c|}
\hline \multirow[b]{2}{*}{ Treatments } & \multicolumn{7}{|c|}{ Morphometric parameters on $50^{\text {th }}$ day } \\
\hline & $\begin{array}{l}\text { Plant height } \\
\text { (cm) }\end{array}$ & $\begin{array}{l}\text { Root length } \\
\text { (cm) }\end{array}$ & $\begin{array}{l}\text { Shoot length } \\
\text { (cm) }\end{array}$ & $\begin{array}{l}\text { No. of } \\
\text { Leaves }\end{array}$ & $\begin{array}{c}\text { No. of. } \\
\text { branches }\end{array}$ & $\begin{array}{c}\text { Root } \\
\text { nodules }\end{array}$ & $\begin{array}{l}\text { No. of } \\
\text { fruits }\end{array}$ \\
\hline T1 & $34 \pm 0.76$ & $10.6 \pm 0.30$ & $24 \pm 0.23$ & 8 & 3 & $6 \pm 1.0$ & 2 \\
\hline $\mathrm{T} 2$ & $34.5 \pm 0.92$ & $10.5 \pm 0.30$ & $24 \pm 0.41$ & 8 & 2 & $5 \pm 1.52$ & 2 \\
\hline $\mathrm{T} 3$ & $35 \pm 0.47$ & $9.5 \pm 0.30$ & $25.5 \pm 0.50$ & 9 & 3 & $6 \pm 1.52$ & 3 \\
\hline $\mathrm{T} 4$ & $33 \pm 0.36$ & $11 \pm 0.26$ & $22 \pm 0.10$ & 8 & 4 & $7 \pm 1.52$ & 2 \\
\hline $\mathrm{T} 5$ & $34 \pm 0.12$ & $12 \pm 0.06$ & $22 \pm 0.06$ & 9 & 3 & $6 \pm 2.0$ & 3 \\
\hline T6 & $34.5 \pm 0.90$ & $12.5 \pm 0.50$ & $22 \pm 0.40$ & 9 & 4 & $5 \pm 1.0$ & 3 \\
\hline $\mathrm{T} 7$ & $31 \pm 0.50$ & $11 \pm 0.50$ & $20 \pm 0.26$ & 7 & 3 & $5 \pm 2.0$ & 3 \\
\hline T8 & $32 \pm 1.07$ & $12 \pm 0.28$ & $20 \pm 0.50$ & 6 & 2 & $6 \pm 1.52$ & 2 \\
\hline T9 & $32.5 \pm 0.78$ & $11 \pm 0.28$ & $21.5 \pm 0.50$ & 6 & 3 & $6 \pm 1.0$ & 1 \\
\hline T10 & $36 \pm 0.91$ & $12 \pm 0.50$ & $24 \pm 0.41$ & 9 & 4 & $20 \pm 2.0$ & 3 \\
\hline T11 & $38.5 \pm 0.56$ & $14 \pm 0.28$ & $24.5 \pm 0.28$ & 10 & 5 & $24 \pm 2.0$ & 5 \\
\hline T12 & $39 \pm 0.52$ & $14 \pm 0.32$ & $25 \pm 0.20$ & 10 & 6 & $28 \pm 3.21$ & 5 \\
\hline T13 & $30 \pm 0.61$ & $10 \pm 0.26$ & $20 \pm 0.41$ & 7 & 5 & $9 \pm 1.52$ & 4 \\
\hline T14 & $32 \pm 0.86$ & $10 \pm 0.50$ & $22 \pm 0.36$ & 8 & 6 & $8 \pm 2.51$ & 3 \\
\hline $\mathrm{T} 15$ & $33 \pm 0.60$ & $11 \pm 0.20$ & $22 \pm 0.40$ & 6 & 4 & $9 \pm 2.51$ & 2 \\
\hline T16 & $30 \pm 0.50$ & $12 \pm 0.32$ & $18 \pm 0.60$ & 7 & 3 & $9 \pm 2.08$ & 3 \\
\hline T17 & $32 \pm 0.40$ & $10 \pm 0.10$ & $22 \pm 0.30$ & 8 & 4 & $6 \pm 1.52$ & 4 \\
\hline T18 & $32.1 \pm 0.26$ & $12 \pm 0.50$ & $20.1 \pm 0.35$ & 7 & 5 & $8 \pm 1.0$ & 3 \\
\hline T19 & $36.4 \pm 0.35$ & $12 \pm 0.18$ & $24.4 \pm 0.17$ & 10 & 5 & $9 \pm 1.0$ & 2 \\
\hline T20 & $38.5 \pm 0.40$ & $15 \pm 0.5$ & $23.5 \pm 0.35$ & 9 & 5 & $25 \pm 1.52$ & 4 \\
\hline $\mathrm{T} 21$ & $37.5 \pm 0.78$ & $12 \pm 0.28$ & $25.5 \pm 0.50$ & 11 & 5 & $25 \pm 1.52$ & 2 \\
\hline Vermicompost & $34 \pm 0.36$ & $12 \pm 0.28$ & $22 \pm 0.41$ & 8 & 5 & $12 \pm 1.0$ & 3 \\
\hline FYM & $36 \pm 0.47$ & $13 \pm 0.28$ & $23 \pm 0.50$ & 8 & 4 & $13 \pm 1.0$ & 3 \\
\hline Control* & $28 \pm 0.87$ & $9 \pm 0.37$ & $19 \pm 0.50$ & 6 & 3 & $7 \pm 1.0$ & 3 \\
\hline \multicolumn{8}{|c|}{ 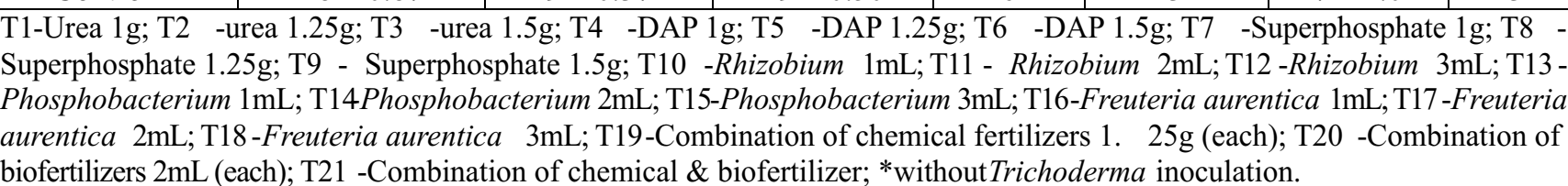 } \\
\hline
\end{tabular}


Table 5. Nutrients contents of Vigna mungo cultivated soil

\begin{tabular}{|c|c|c|c|c|c|c|c|c|c|c|c|c|}
\hline \multirow[b]{2}{*}{ Treatment } & \multicolumn{11}{|c|}{ Parameters } & \multirow{2}{*}{$\begin{array}{c}\text { Fungal } \\
\text { propagules } \\
/ \mathrm{g}\end{array}$} \\
\hline & pH & $\underset{\left(\mathrm{dSm}^{-1}\right)}{\mathbf{E C}}$ & $\begin{array}{l}\mathrm{CaCO}_{3} \\
(\mathrm{mg} / \mathrm{kg})\end{array}$ & $\begin{array}{c}\mathbf{N} \\
(\mathrm{kg} / \mathrm{ha})\end{array}$ & $\begin{array}{c}\mathbf{P} \\
(\mathrm{kg} / \mathrm{ha})\end{array}$ & $\begin{array}{c}\mathbf{K} \\
(\mathrm{kg} / \mathrm{ha})\end{array}$ & $\begin{array}{c}\mathbf{F e} \\
(\mathrm{ppm})\end{array}$ & $\begin{array}{c}\text { Mn } \\
(\mathrm{ppm})\end{array}$ & $\begin{array}{c}\mathbf{Z n} \\
(\mathrm{ppm})\end{array}$ & $\begin{array}{c}\mathbf{C u} \\
(\mathrm{ppm})\end{array}$ & $\begin{array}{l}\mathrm{OC} \\
(\%)\end{array}$ & \\
\hline $\begin{array}{l}\text { Control pot (Without any } \\
\text { supplements) }\end{array}$ & 8.1 & 1.7 & Medium & 50.0 & 4.0 & 143.8 & 2.70 & 2.25 & 0.90 & 0.80 & 0.27 & 32 \\
\hline $\begin{array}{l}\text { Soil }+ \text { Rhizobium }+ \\
\text { Trichoderma }\end{array}$ & 7.2 & 0.54 & High & 85.6 & 10.8 & 368 & 2.13 & 2.89 & 1.56 & 0.42 & 0.38 & 47 \\
\hline $\begin{array}{l}\text { Soil + Phophobacterium }+ \\
\text { Trichoderma }\end{array}$ & 7.3 & 0.72 & High & 68.9 & 14.5 & 374 & 3.02 & 2.87 & 1.32 & 0.50 & 0.42 & 38 \\
\hline $\begin{array}{l}\text { Soil }+ \text { Frateuria aurantia }+ \\
\text { Trichoderma }\end{array}$ & 7.5 & 0.67 & High & 70.5 & 14.1 & 380 & 2.80 & 3.10 & 1.24 & 0.52 & 0.44 & 42 \\
\hline $\begin{array}{l}\text { Soil + Farm yard manure + } \\
\text { Trichoderma }\end{array}$ & 7.2 & 0.77 & Medium & 80.2 & 14.3 & 365 & 2.74 & 2.80 & 1.09 & 0.43 & 0.36 & 36 \\
\hline $\begin{array}{l}\text { Soil + Vermicompost + } \\
\text { Trichoderma }\end{array}$ & 7.3 & 0.58 & Medium & 73.5 & 12.9 & 350 & 2.68 & 2.64 & 0.99 & 0.58 & 0.39 & 40 \\
\hline Soil + Urea + Trichoderma & 8.1 & 0.55 & High & 61.2 & 12.4 & 340 & 2.40 & 2.18 & 0.89 & 0.80 & 0.32 & 22 \\
\hline $\begin{array}{l}\text { Soil + Super phosphate + } \\
\text { Trichoderma }\end{array}$ & 8.3 & 0.52 & High & 52.0 & 15.1 & 322 & 3.01 & 2.14 & 0.78 & 0.70 & 0.34 & 26 \\
\hline Soil + DAP + Trichoderma & 8.0 & 0.47 & High & 70.1 & 15.2 & 311 & 2.85 & 2.24 & 0.91 & 0.54 & 0.28 & 24 \\
\hline
\end{tabular}

metals like $\mathrm{Zn}$ and $\mathrm{Cu}$ and $\mathrm{OC}$ content was reduced due to utilization of plants. Further, the population of mycoflora of the biofertilizers treated soil also increased when compared to the control and chemical fertilizers treated soil (Table 5). Two-way analysis of variance (ANOVA) test was examined between the treatments and nutrient contents of Vigna mungo treated soil, and the results are presented in table 6 . A positive significant $(p<0.05)$ variation was observed between the nutrient parameters, but there was no significance between the treatments of Vigna mungo treated soil.

Table 6. Two-way ANOVA for the treatments and nutrient content of Vigna mungo treated soil.

\begin{tabular}{|l|c|c|c|c|c|}
\hline \multicolumn{1}{|c|}{ Source of Variation } & SS & $\boldsymbol{d f}$ & $\boldsymbol{M S}$ & $\boldsymbol{F}$ & $\boldsymbol{P}$-value \\
\hline Rows (Treatments) & 5609.41 & 8 & 701.1763 & 1.442245 & 0.192047 \\
\hline Columns (Nutrient parameters) & 851564.2 & 10 & 85156.42 & 175.1577 & $4.65 \mathrm{E}-50^{*}$ \\
\hline Error & 38893.61 & 80 & 486.1701 & & \\
\hline Total & 896067.2 & 98 & & & \\
\hline${ }^{*} p<0.05$ is statistically significant \\
\hline
\end{tabular}

\section{DISCUSSION}

The fertility of the soil and water mass is determined by the distribution of nutrients (Bragadeeswaran et al., 2007). Soil has many physical and chemical properties, some are changeable, while others are difficult to adjust. Texture, structure, drainage, and organic matter content are the physical properties. Chemical properties including cation exchange capacity and $\mathrm{pH}$ generally affect microbial as well as plant growth (Cholarajan and Vijayakumar, 2013). The present study reported the diversity of 8 different soil fungal genera among 1018 isolated fungi from cultivated land soil samples. The frequency of soil fungi from 38 locations of 10 different districts of Tamilnadu revealed that Aspergillus niger and T. harzianum were the most frequently documented fungi in all the soil samples (Table 2). In line with the present observations, Naveenkumar et al. (2011) and Chandrashekar et al. (2014) also reported the predominance of Aspergillus spp. from agricultural and non-agricultural soils of Shimoga and Mysore districts of Karnataka, respectively. Presently, to begin with foldscope was used so as to identify the soil fungi the microscopic details of which was subsequently confirmed by using conventional light microscope. The uniformity in primary observations made and subsequent identification of all the test fungi using foldscope and light microscope brought out the reliability of the origami device - foldscope for making such studies. Using foldscope similar such studies have been made for the identification of Colletotrichum gloeosporoides, C. capsici and Fusarium oxysporum by Gurjar and Kanade (2020), Penicillium sp. and Aspergillus sp. by Prarthana and Narayana (2020); mycorrhizal fungi by Nivedha et al. (2019) and Sabarinathan et al. (2019). When physico-chemical properties of the soil were studied, the $\mathrm{pH}$ of the soil samples was found to vary from neutral to slightly alkaline in nature. As compared, Pešaković et al. (2009) documented acidic ( $\mathrm{pH}$ 5.9) nature of the alluvial soil in Serbia. Likewise, Nakhro and Dkhar, (2010) documented varied levels of $\mathrm{pHs}$ in control $(5.310 \pm 0.17)$, organic $(5.120 \pm 0.14)$ and inorganic $(5.350 \pm 0.13)$ field soil, respectively. However, Lazcano et al. (2012) reported slight acidic nature of the soil $\mathrm{pH}(6.6 \pm 0.02)$. Although it is a well established fact that most of the fungal populations prefer acidic $\mathrm{pH}$ ranges, but during the present investigations the documented fungal taxa were isolated from the soil samples having neutral to slight alkaline $\mathrm{pH}$ range. In general, it is a known fact that the diversity of native microbial population of a particular ecosystem is regulated by the physical, chemical as well as biological factors. During the present investigations, no positive correlation was observed between the biological and physico-chemical factors of the soil. In a related work, statistical significance between the soil microorganisms and soil nutrients has already been reported using correlation co-efficient studies by Vijayakumar et al. (2007).

To understand the impact of use of chemical fertilizers on the occurrence and survivability of population of indigenous microflora in the soil three chemical fertilizers were tested. The mycelial dry weights of the A. niger and T. harzianum present in the soil was significantly reduced when higher concentration of urea and super phosphate combination was broadcasted in the soil. This may be due to the toxic effect of urea when used in higher concentration. This observation is in 
conformity with the earlier such reports by Veverka et al. (2007) and Karthika and Vijayakumar (2018). Initial propagule densities of Trichoderma spp. were found to be higher in soils amended with swine manure and cotton-gin trash (CGT) in comparison to the soils amended with ryevetch or synthetic fertilizer. Bulluck III and Ristaino (2002) also documented increased propagule density of the Trichoderma spp. over a period in the soils when amended with synthetic fertilizers. Thus, the results of the present study are in conformity with the earlier reports by Bulluck III and Ristaino (2002). Noticeably, the tolerance capability of the test fungi differed between the methods employed and media used. In the solid media, the radial growth of the test fungi was significantly inhibited when the medium was added with urea, whereas mycelial dry weights of the test fungi was reduced at higher concentration of urea and super phosphate combination.

Recently, species of Trichoderma have been effectively used as biostimulant for the growth and development of a wide variety of plants (Bhardwaj et al., 2014). Reports also suggested that Trichoderma produces plant growth promoting phytohormones such as indole acetic acid (IAA) and their analogous (Vinale et al., 2012), vitamins, enzymes leading to plant growth, organic acids in rhizosphere such as gluconic, citric, and/or fumaric acids which decrease the soil $\mathrm{pH}$ (Harman et al., 2004). Hence, the usage of plant growth promoting Trichoderma species is becoming one of the important aspects of sustainable eco-friendly approach in agriculture. During the present study, the predominantly documented soil fungus of the studied region T. harzianum was selected and incorporated into all the treatments of pot culture experiment, and also it was found that all the plant growth related parameters were higher in fertilizers treated pots. Further, the physico-chemical parameters of the treated soil when analyzed, it was observed that the $\mathrm{pH}$ of biofertlizers treated soil changed to neutral condition. In addition, positive significant $(\mathrm{p}<0.05)$ variation was observed between the nutrient's parameters of Vigna mungo, but there was no significance noted between the treatments. Propagule densities of microbial species including total cultivable fungi, thermophilic microorganisms, Phytophthora and Pythium spp., and numbers of sclerotia were not significantly affected by treatments over the course of the experiment (Bulluck III and Ristaino, 2002). In contrast to this, Islam et al. (2017) reported that most of the parameters were significantly increased after treatment except $\mathrm{N}$ and $\mathrm{S}$ contents. Mathivanan et al. (2014) also reported that most of the morphometric data of the groundnut (Arachis hypogeaea L.) and nutrient parameters of the soil increased significantly (at $\mathrm{P}<0.05$ ). These changes may be due to the presence of microbial population which is involved in the biogeochemical cycles and addition of NPK chemical fertilizers.

Generally, age and number of leaves and area of leaf surface accounts for the increase in the quantity of chlorophyll content of the plants. In the present study, the chlorophyll a and chlorophyll $\mathrm{b}$ were estimated from the test plant obtained from pot culture experiment, and maximum chlorophyll contents was found in the test plant treated with Rhizobium bioinoculant. Similarly, Hajieghrari and Mohammadi (2016) reported that no significant reduction was found in the chlorophyll content of the Trichoderma fortified wheat plants.

\section{CONCLUSION}

The findings of the present study brought out that the $T$. harzianum and $A$. niger were the most predominant fungal taxa of the studied stations. Further, it was found that among three nitrogen fertilizers tested, urea had the most significant effect on the growth of T. harzianum and Aspergillus niger even at lower concentration. However, the impacts of chemical fertilizers on soil microbial processes and nutrient cycling could be influenced by different factors as well such as crop rotation, soil type, microbial varieties and compost properties.

\section{ACKNOWLEDGEMENTS}

This work was supported by grants from the Department of Biotechnology, New Delhi, Government of India (BT/IN/Indo-US/ Foldscope/39/2015 dated 20.04.2018). The authors are grateful to K. Panneer Selvam and Suresh S.S. Raja for comments and suggestions on the manuscript and C. Muthukumar for assistance with the statistical analysis.

\section{REFERENCES}

Baishya, K., 2015. Impact of agrochemicals application on soil quality degradation - a Review. Int. J. Sci. Technol. Mgmt. 4(1): 220-228.

Bhardwaj, D., Ansari, M.W., Sahoo, R.K. and Tuteja, N. 2014. Biofertilizers function as key player in sustainable agriculture by improving soil fertility, plant tolerance and crop productivity. Microb Cell Fact. 13: 66.

Bragadeeswaran, S., Rajasegar, M., Srinivasan, M. and Kanaga Rajan, U. 2007. Sediment texture and nutrients of Arasalar estuary, Karaikkal, southeast coast of India. J. Environ. Biol. 28: 237 - 240.

Bridge, P. and Spooner, B. 2001. Soil fungi: diversity and detection. Plant Soil 232: 147-154.

Bulluck III, L.R. and Ristaino, J.B. 2002. Effect of Synthetic and Organic Soil Fertility Amendments on Southern Blight, Soil Microbial Communities, and Yield of Processing Tomatoes. Phytopathology 92(2): 181189.

Chandrashekar, M.A., Soumya Pai, K. and Raju, N.S. 2014. Fungal diversity of rhizosphere soils in different agricultural fields of Nanjangud taluk of Mysore district, Karnataka, India. Int. J. Curr. Microbiol. App. Sci. 3 (5): 559-566.

Cholarajan, A. and Vijayakumar, R. 2013. Analyses of physical and chemical parameters of the rhizosphere soils from Thanjavur district, Tamil Nadu, India. $J$. Ener. Environ. Sci. Photon 127: 219-223.

Cybulski, J.S., Clements, J. and Prakash, M. 2014. Foldscope: Origami-based paper microscope. PLOS ONE 9: 
e98781. Https://doi.org/10.1371/journal.pone. 0098781.

Eaton, A.D., Clesceri, L.S., Greenberg, A.E. and Franson, M.A.H. 2005. Standard methods for the examination of water and wastewater. American Public Health Association 21: 1600.

Gillman, J.C. 1957. A Manual of Soil Fungi. 2nd Edn., The Lowa State University Press, Ames, Lowa, 450.

Gnanasekaran, P., Mohamed, S.S. and Panneerselvam, A. 2015. Isolation and identification of soil mycoflora in banana field at Manachanallur, Tiruchirappalli Dt., Tamil Nadu, India. Int. J. Curr. Microbiol. App. Sci. 4: 729-740.

Gurjar, G.S. and Kanade, M.G. 2019. A glimpse into the plant world through foldscope (Ed.: Sharma, A.D.). National Press Associates, New Delhi, pp. 65-75.

Hafez, A. S.I.I. 1982. Cellulose-decomposing fungi of desert soils in Saudi Arabia, Mycopathologia 78 (2): 73-78.

Hajieghrari, B. and Mohammadi, M. 2016. Growthpromoting activity of indigenous Trichoderma isolates on wheat seed germination, seedling growth and yield. Aus. J. Corp. Sci. 10: 1339-1347.

Harman, G.E., Howell, C.R., Viterbo, A., Chet, I. and Lorito, M. 2004. Trichoderma species - opportunistic, avirulent plant symbiont. Nat. Rev. Microbiol. 2: 4356.

Islam, M.A., Islam, S., Akter, A., Rahman, M.H. and Nandwani, D. 2017. Effect of organic and inorganic fertilizers on soil properties and the growth, yield, and quality of tomato in Mymensingh, Bangladesh. Agriculture 7: 1-7.

Karthika, K. and Vijayakumar, R. 2018. Occurrence and fertilizer tolerance capacity of soil fungi isolated from cultivated soils of Perambalur district, Tamilnadu. Abstr. In: Int. Symp. Fungal Biol.: Adv., Applications Conservation \& 45th Annual Meeting of Mycological Society of India (MSI), Nov. 19-21, 2018, Pune, Maharashtra.

Karthika, K., Suresh S.S. Raja, Bhuvaneshwari, B., Vinoth, M. and Vijayakumar, R. 2019. Application of foldscope as a modernized tool for the diversity of fungi and its tolerance against synthetic fertilizers. Proc. Int. Seminar Recent Trends Biol. Sci. : 62-76.

Khattabi, N., Ezzahiri, B., Louali, L. and Oihabi, A. 2004. Effect of nitrogen fertilizers and Trichoderma harzianum on Sclerotium rolfsii. Agronomie 24: 281-288.

Kumawat, T.K., Sharma, A. and Bhadauria, S. 2016. Influence of liquid culture media, temperature and hydrogen ion concentration on the growth of mycelium and sporulation of Arthroderma multifidum. Int. J. Pharma. Sci. Rev. Res. 41: 136141.
Latha, P., Jeyaraman, S. and Prabakaran, R. 2014. Study effects of microbial and chemical fertilizer on yield omponents in brinjal (Solanum melongena Linn.) C.Var CO-2. Int. J. Curr. Microbiol. App. Sci. 3: 817822.

Lazcano, C., Gómez-Brandón, M., Revilla, P. and Domínguez, J. 2012. Short-term effects of organic and inorganic fertilizers on soil microbial community structure and function. A field study with sweet corn. Biol Fertil Soils 49(6): DOI 10.1007/s00374-012-0761-7.

Lin, K.H., Huang, M.Y., Huang, W.D., Hsu, M.H., Yang, Z.W. and Yang, C.M. 2013. The effects of red, blue, and white light-emitting diodes on the growth, development, and edible quality of hydroponically grown lettuce (Lactuca sativa L. var. capitata). Sci. Hortic. 150: 86-91.

Mathivanan, S., Chidambaram, A.L.A., Sundramoorthy, P., Baskaran, L. and Kalaikandhan, R., 2014. Effect of combined inoculations of plant growth promoting rhizobacteria (PGPR) on the growth and yield of groundnut (Arachis hypogaea L.). Int. J. Curr. Microbiol. App. Sci. 3(8): 1010-1020.

Nakhro, N. and Dkhar, M.S, 2010. Impact of organic and inorganic fertilizers on microbial populations and biomass carbon in paddy field soil. J. Agronomy 9(3): 102-110.

Naveenkumar, K.J., Thippeswamy, B., Thirumalesh, B.V., Pradeepa, K. and Venkatesh, 2011. Comparative study of fungal diversity in the agricultural soil and non-agricultural soil of Bhadravathi taluk, Shimoga district, Karnataka, India. J. Res. Biol. 1: 129-134.

Nivedha. D, Gokilavani, R. and Rehana Banu, H., 2019. Exploration of arbuscular mycorrhizal fungi (AMF) in the rhizosphere of five varieties of Cocos nucifera l. during summer season in Pollachi Taluk, Coimbatore district, Tamilnadu, India (Ed.: Sharma, A.D.). National Press Associates, New Delhi, pp. 193-200.

Pešaković, M., Đukić, D., Mandić, L. and Miletić, R. 2009. Effect of NPK fertilizer on the growth of saprophytic fungi in alluvial soil. J. Central European Agri. 10(4): 449-454.

Prarthana. J. and Narayana, 2019. Capturing diverse, impressive images using foldscope. (Ed.: Sharma, A.D.). National Press Associates, New Delhi, pp.175-187.

Ratna Kumar, P.K., Shiny Niharika, P. and Hemanth, G. 2017. Impact of fungicides on the growth and distribution of soil mycoflora in agriculture fields at Narasannapeta. Int. J. Sci. Res. 6: 2337-2347.

Sabarinathan K.G., Gomathy, M., Arun Kumar, D. and Kannan, R. 2019. Foldscope: an innovative tool to study agriculturally important microbes (Ed.: Sharma, A.D.). National Press Associates, New 
Delhi, pp. 205-211.

Veverka, K., Stolcova, J. and Ruzek, P. 2007. Sensitivity of fungi to urea, ammonium nitrate and their equimolar solution UAN. Plant Protect. Sci. 43: 157-164.

Vijayakumar, R., Muthukumar, C., Thajuddin, N., Panneerselvam, A. and Saravanamuthu, R. 2007. Studies on the diversity of actinomycetes in the Palk
Strait region of Bay of Bengal, India. Actinomycetologic 21:59-65.

Vinale, F., Sivasithamparam, K., Ghisalberti, E.L., Ruocco, M., Wood, S. and Lorito, M. 2012. Trichoderma secondary metabolites that affect plant metabolism. Nat. Prod Commun. 7: 1545-1550. 